\title{
BMJ Open Trajectories of mid-life to elderly adulthood BMI and incident hypertension: the China Health and Nutrition Survey
}

\author{
Qi Zeng, Lin Sun, Qing Zeng
}

To cite: Zeng Q, Sun L, Zeng Q. Trajectories of mid-life to elderly adulthood BMI and incident hypertension: the China Health and Nutrition Survey. BMJ Open 2021;11:e047920. doi:10.1136/ bmjopen-2020-047920

- Prepublication history and additional supplemental material for this paper are available online. To view these files, please visit the journal online (http://dx.doi.org/10.1136/ bmjopen-2020-047920)

Received 15 December 2020 Accepted 18 June 2021

Check for updates

(C) Author(s) (or their employer(s)) 2021. Re-use permitted under CC BY-NC. No commercial re-use. See rights and permissions. Published by BMJ.

Department of Epidemiology and Health Statistics, School of Public Health and Management, Chongqing Medical University, Chongqing, China

Correspondence to Professor Qing Zeng; zengqing@cqmu.edu.cn

\section{ABSTRACT}

Objectives This study is designed to identify different body mass index (BMI) trajectories of individuals aged 40-70 years and test the effect of distinct BMI trajectories on incident hypertension.

Design The accelerated longitudinal design was used for this study.

Methods The study drew data from the third to ninth China Health and Nutrition Surveys (CHNS), and 4697 participants were included between 1991 and 2015. As analysed, three distinct individual BMI trajectories were identified by the latent class growth mixed model (LCGMM). Then, BMI values and BMI slopes were worked out through calculation with LCGMM trajectory parameters and their primary derivatives, respectively. Later, Cox proportional hazard models were applied to examine BMI values and slopes, and find out the relationship between the said predicted data and incident hypertension for different classes.

Results Three different trajectory classes were identified, that is, low-stable class $(n=3711)$, sharp-increasing class $(n=282)$ and high-stable class $(n=704)$. Compared with the low-stable class, the adjusted HRs $(95 \% \mathrm{Cl})$ were 1.321 (1.119 to 1.559) and 1.504 (1.322 to 1.711) for the sharpincreasing class and the high-stable class, respectively. The HR $(95 \% \mathrm{Cl})$ for $\mathrm{BMI}$ values rose from 1.081 (1.030 to 1.135$)$ to 1.221 (1.171 to 1.273$)$ while the $\mathrm{HR}(95 \% \mathrm{Cl})$ for BMI slopes dropped from 1.154 (1.100 to 1.211) to 0.983 (0.943 to 1.025). That is, the HR for BMI slopes were higher than that for BMl values for the class aged $40-47$ years.

Conclusion These findings suggest that the calibrated BMI trajectories for the period from mid-life to elderly adulthood have a significant effect on the risk of incident hypertension. The period from age 40 to 47 years is critical and has positive implications for the early prevention of hypertension.

\section{INTRODUCTION}

As the economy develops and the urbanisation evolves, more and more Chinese adults are beginning to suffer from hypertension. ${ }^{1-3}$ For this, obesity or increased body mass index
Strengths and limitations of this study

The data used in this study were from a large national survey conducted from 1991 to 2015 .

- Latent class growth mixed models were used to characterise different individual body mass index (BMI) trajectories.

- This study explored BMI values and slopes at distinct ages (40-70 years) for predicting the risk of hypertension in later life.

- Some patients with hypertension were recognised by self-report, and some cases of incident hypertension may be overlooked.

(BMI) is identified as an important individual risk factor. $^{4-6}$ As concluded by numerous researchers, both weight change and BMI value were related to the occurrence of hypertension. ${ }^{78}$ However, most studies are cross-sectional or cohort studies based on a limited number of BMI measurements, not taking into account the dynamic trends in BMI changes over the life process.

For a better understanding of the correlation between BMI and the risk of hypertension in later life, researchers turn to the trajectory of BMI changes as BMI trajectories reflect the dynamic changes of BMI in a certain period. ${ }^{9}$ A few longitudinal studies have found that BMI trajectories in children, adolescents and early adults are associated with the elevation of blood pressure and incident hypertension. ${ }^{10-14}$ Yuan et al once reported three different BMI trajectories in early life that are correlated with cardiometabolic risk. ${ }^{15}$ Nevertheless, a few studies have confirmed BMI trajectory classes and their relationship with the incidence of hypertension from mid-life to elderly adulthood. In this study, we assumed that participants in distinct BMI trajectories were at a different risk of incident hypertension during their mid-life to elderly adulthood, and from 
the increased rate of BMI (BMI slope) then, we may predict the onset of hypertension.

Using China Health and Nutrition Survey (CHNS) data from 1991 to 2015, this study aims to characterise different BMI trajectories during the mid-life to elderly adulthood (40-70 years), examine the relationship between BMI trajectory classification and incident hypertension, and explore the relation between BMI slopes and incident hypertension.

\section{METHODS}

\section{Data source and participants}

Data for the study were obtained from 9 waves of the CHNS during 1991-2015, which is an ongoing open survey that collected data in 10 visits from 1989 to 2015, conducted by the Carolina Population Center and the National Institute of Nutrition and Health, and at last covered 15 states and cities (initially 9). In this study, a multistage random cluster sampling method ${ }^{16}$ was adopted to extract research objects. For your reference, data are available at the CHNS website (https://www.cpc.unc.edu/projects/ china/data/datasets/data_downloads).

As released by the data, 13111 participants aged 40-70 years without hypertension at baseline were included, and participants without BMI values or subject to less than three follow-up investigations were excluded. After the exclusion, we selected 4697 participants, who were visited 4.48 times (range: $3-9$ times) in this study. That is, the number of follow-up individuals in 1991, 1993, 1997, 2000, 2004, 2006, 2009, 2011 and 2015 are 1486, 1747, 2392, 2659, 2882, 2877, 2862, 2372 and 1744, respectively. The average number of follow-up years is 11.51 (range: 5-24 years). The data after incident hypertension were removed in analyses.

\section{Data collection and definition}

For CHNS data collection, all investigators and inspectors were well-trained in advance. Besides, data were collected under the supervision of the University of North Carolina at Chapel Hill, the Chinese Center for Disease Control and Prevention and the China-Japan Friendship Hospital. ${ }^{17}$

\section{Outcome: incident hypertension}

Most subjects went through all standardised tests, including the testing of systolic blood pressure (SBP) and diastolic blood pressure (DBP). The blood pressure was measured on the right arm by trained health technicians using a mercury sphygmomanometer with a suitable cuff. In the process, three measurements were taken when the subject sat still for $10 \mathrm{~min}$. Readings of SBP at the first pulse sound and DBP at the last pulse sound were recorded. To reduce the impact of measurement errors, the average value of three measurements was used for the analyses. Then, the onset of hypertension was defined when DBP $\geq 90 \mathrm{~mm} \mathrm{Hg}$ and/or SBP $\geq 140 \mathrm{~mm} \mathrm{Hg}^{18}$ or through selfreport, and participants without hypertension at baseline was confirmed to have incident hypertension as the said diagnosis criteria were met in follow-up examination. ${ }^{19}$

\section{Time variable: age}

The accelerated longitudinal design (ALD) ${ }^{20}$ was used for studying the BMI development from mid-life to elderly adulthood, taking the age as the timeline. As confirmed by some researches, ALD is superior in researching age-related changes over time as it can group multiple cohorts. ${ }^{21}$ Compared with the single-cohort design, the data might be less sensitive to the dropout in the ALD. ${ }^{20}$

\section{Trajectory variable: BMI}

Participants came with light clothing and no shoes. Their weight and height were measured with a portable tachometer and a calibrated scale, respectively. The participants wore light clothing and no shoes. The measurement error remains at $0.1 \mathrm{~cm}$ and $0.1 \mathrm{~kg}$, respectively. Then, the mean value of the two measurements in one investigation was computed as the record value. ${ }^{22}$ It should be noted that the height and weight data were collected by well-trained operators according to the standard procedures of the WHO. BMI $\left(\mathrm{kg} / \mathrm{m}^{2}\right)$ was computed by dividing weight $(\mathrm{kg})$ by height $(\mathrm{m})$ squared. One with a BMI between 24 $\mathrm{kg} / \mathrm{m}^{2}$ and $27.9 \mathrm{~kg} / \mathrm{m}^{2}$ was defined to be overweight, and obese for those at $28 \mathrm{~kg} / \mathrm{m}^{2}$ or more. ${ }^{23}$

\section{Covariates}

In this study, some potential covariates could affect the relationship between BMI trajectory classes and incident hypertension. The demographical factor, health-related behaviour factor and the blood pressure at baseline were associated with incident hypertension.

Sociodemographic factors ${ }^{24}$ cover age, gender, urbanrural residence, marital status, working status and education level. Health-related behaviour factors cover smoking and alcohol drinking. All factors were collected through CHNS personal questionnaire. In the questionnaire, age was calculated in years to 0 decimal points; question T2 was set for the nature of urban-rural residence; question U25 was to ask for smoking (have you ever smoked cigarettes (including hand-rolled or device-rolled)?), question U40 for alcohol drinking (do you drink beer or any other alcoholic beverage?), question A8 for marital status (what is your marital status?), question B2 for working status (are you working?) and question A12 for the education level (what is your highest education degree?). The options of being never married, divorced, widowed and separated were combined as others in marital status. The education level was divided into three categories: (1) have not attended a school or finished the primary school, (2) junior high school or below and (3) senior high school or above.

\section{Statistical analysis}

Descriptive and univariate analysis

Shapiro-Wilk test was applied to test continuous variables for normality. Characteristics were summarised by using the median (IQR) for continuous variables and the count 
(proportion) for discrete variables. Mann-Whitney U test and Kruskal-Wallis $\mathrm{H}$ test (for continuous variables) and $\chi^{2}$ test (for discrete variables) were used to compare the characteristics of distinct classes.

Estimation of individual BMI trajectories, trajectory classification, values and slopes

Latent class growth mixed models (LCGMMs) ${ }^{25}$ were applied to characterise distinct individual BMI trajectories. As gender has an impact on trajectory classification, it was taken as a covariable of trajectory fitting, and 15 LCGMMs with distinct shapes, including linear, quadratic and cubic term parameters, were tested through trajectory fitting with BMI measured at least three times between 1991 and 2015. Repeated analysis of trajectories of two to five classes was performed and the best model was identified with the same starting values calculated from the model for class 1 . The best model was determined as per the following criteria: ${ }^{25} 26$ (1) the Bayesian information criterion (BIC) value was relatively small, with a decrease of at least 20 ; (2) the average posterior probability of assignment (APPA) $\geq 0.65$; (3) the entropy (the relative entropy value) $\geq 0.50$; and (4) the proportion of each category was greater than $1 \%$. Then the best model was worked out as the quadratic trajectory of the three classes for which the model expression was presented as:

$$
\left.B M I_{i j}\right|_{C_{i}=g}=\left(v_{0 g}+u_{0 i g}\right)+\left(v_{1 g}+u_{1 i g}\right) t i m e+\left(v_{2 g}+u_{2 i g}\right) t i m e^{2}+\varepsilon_{i j}
$$

where the vector $v=\left(v_{0 g}, v_{1 g}, v_{2 g}\right)$ is the fixed effect parameter of class $g$, and the vector $u=\left(u_{0 i g}, u_{1 i g}, u_{2 i g}\right)$ is the random effect parameter of individual $i$ in class $g$, while time $=$ age -40 and $\varepsilon_{i j}$ is a random error term.

The fixed effect parameter reflects the average trajectory of each class, and the random effect parameter reflects the difference between individual trajectories and the average trajectory of individuals. By combing with fixed effect and random effect parameters, 4697 different individual trajectory curves were generated. Based on the said individual trajectory curves and their first derivatives, both BMI values and slopes were worked out. To be specific, BMI slopes and BMI values were calculated for the age of 40-70 years, one per year, and were standardised with z-score.

Individual BMI trajectory classification and modelpredicted BMI values and slopes were used as influential factors in the subsequent analyses of incident hypertension.

\section{Individual BMI trajectory classification and incident hypertension}

Cox proportional hazard models were applied to study the relationship between the BMI trajectory classification and the onset of hypertension and factors, including gender, age, education level, smoking, alcohol drinking, DBP and SBP, and marital status was adjusted.

Individual model-predicted BMI values, slopes and incident hypertension

Cox proportional hazard models were adopted to test the relationship between the model-predicted BMI values and slopes and incident hypertension at different ages. HRs (95\% CI) of model-predicted BMI values and slopes for the incidence of hypertension were estimated, being adjusted for gender, smoking, alcohol drinking, working status, urban-rural residence, marital status and education level. In addition, BMI values and BMI slopes were adjusted for each other in Cox regression models.

\section{Statistical software}

$\mathrm{R}$ (V.3.6.2) was used for data management and statistical analyses, and the lcmm (V.1.8.1) package was used to estimate LCGMMs, in which the significance level was set as $\alpha=0.05$.

\section{RESULTS}

\section{Descriptive and univariate analyses}

Table 1 sums up the overall distribution of research variables and those in follow-up incident hypertension. Gender, alcohol drinking, working status, education level, BMI, age, SBP and DBP were significantly distinct among normotensives and hypertensives. During the follow-up, 1954 (41.60) incident primary hypertensives were identified. Hypertensives had higher BMI, age, SBP, DBP and follow-up time, and higher proportion of men, drinkers, smokers, employed and living in rural areas than normotensives.

Online supplemental table S1 shows the results of the 15 LCGMMs fitting process. In the LCGMM, BMI is a dependent variable, time (time $=$ age -40 ) is an independent variable, the and gender is a class membership covariate. Since class 4 accounts for a low proportion $(0.51 \%)$, the quadratic trajectory model of five classes was excluded. According to the trajectory model selection criteria, the model of quadratic curves for three classes with the lowest BIC (85 004.676), a higher entropy (0.581), APPA $(0.85 / 0.65 / 0.73)$ and percentage of class memberships $(79.01 \% / 6.00 \% / 14.99 \%)$ was selected.

Figure 1 shows the three different trajectory classes of BMI. From the perspective of the way the trajectories changed, BMI trajectory classes were labelled as the lowstable $(n=3711)$, the sharp-increasing $(n=282)$ and the high-stable $(\mathrm{n}=704)$. Online supplemental table S2 gives the curve parameters with fixed and random effects in the three trajectory classes. The trajectory parameters $(v, u$ and $\beta)$ were significantly distinct from $0 \quad(\mathrm{p}<0.05)$ except for the coefficient of quadratic (sharp-increasing). The average trajectory expressions of the three classes were as follows:

Low-stable class:BMI $=21.130+0.069$ time -0.001 time $^{2}$

Sharp-increasing class: $\mathrm{BMI}=22.228+0.199$ time $^{2}+0.002$ time $^{2}$

High-stable class:BMI $=25.919+0.075$ time -0.003 time $^{2}$

In the low-stable class, BMI was maintained at about $22 \mathrm{~kg} / \mathrm{m}^{2}$ for the age from $40-70$ years. In the sharpincreasing class, BMI increased rapidly from $22.23 \mathrm{~kg} /$ $\mathrm{m}^{2}$ at age 40 years to $29.90 \mathrm{~kg} / \mathrm{m}^{2}$ at age 70 years. In the high-stable class, BMI remained stable at about $26 \mathrm{~kg} / \mathrm{m}^{2}$ during the age $40-70$ years $($ time $=$ age -40$)$. 
Table 1 Baseline characteristics of hypertensive events in follow-ups.

\begin{tabular}{|c|c|c|c|c|}
\hline & Total $(n=4697)$ & Normotensives $(n=2743)$ & Hypertensives $(n=1954)$ & $P$ value \\
\hline Men, n (\%) & 2085 (44.39) & $1175(42.84)$ & $910(46.57)$ & 0.011 \\
\hline Smoking, n (\%) & $1614(34.46)$ & $890(32.54)$ & 724 (37.15) & 0.001 \\
\hline Drinking, n (\%) & $1728(37.03)$ & 947 (34.78) & $781(40.17)$ & $<0.001$ \\
\hline Married, n (\%) & $4420(94.48)$ & $2575(94.18)$ & $1845(94.91)$ & 0.286 \\
\hline Job, n (\%) & $3836(81.79)$ & $2204(80.44)$ & 1632 (83.69) & 0.004 \\
\hline Education, n (\%) & & & & $<0.001$ \\
\hline Primary school or no & $2537(54.55)$ & $1334(49.21)$ & $1203(62.01)$ & \\
\hline Middle school & $1190(25.59)$ & $756(27.89)$ & $434(22.37)$ & \\
\hline High school & $924(19.87)$ & $621(22.91)$ & $303(15.62)$ & \\
\hline Urban, n (\%) & $3204(68.21)$ & $1796(65.48)$ & $1408(72.06)$ & $<0.001$ \\
\hline $\mathrm{BMI}, \mathrm{kg} / \mathrm{m}^{2}$ & $22.02(3.73)$ & $21.76(3.58)$ & $22.35(3.79)$ & $<0.001$ \\
\hline Age, years & $43.00(8.00)$ & $43.00(8.00)$ & $44.00(9.00)$ & $<0.001$ \\
\hline SBP, mm Hg & $111.33(15.00)$ & $110.00(18.17)$ & $116.00(13.00)$ & $<0.001$ \\
\hline DBP, mm Hg & 74.67 (10.00) & 73.33 (11.33) & 76.00 (10.00) & $<0.001$ \\
\hline Follow-up, year & $11.00(8.00)$ & $11.00(8.00)$ & $9.00(7.00)$ & $<0.001$ \\
\hline
\end{tabular}

Estimation of individual BMI trajectories and trajectory classification

The number (\%) of missing value was $13(0.28 \%)$ for smoking, $30(0.64 \%)$ for alcohol drinking, $19(0.40 \%)$ for marital status, 7 (0.15\%) for working status and $46(0.98 \%)$ for education level

$\mathrm{BMI}$, body mass index; DBP, diastolic blood pressure; SBP, systolic blood pressure.

Individual BMI trajectory classification and incident hypertension Online supplemental table S3 summarises the research characteristics at baseline by BMI trajectory classes (LCGMM result). The proportion of incident hypertension was significantly distinct among the three BMI trajectory classes $(\mathrm{p}<0.001)$. Significant differences in gender, smoking, alcohol drinking, employment, education level,

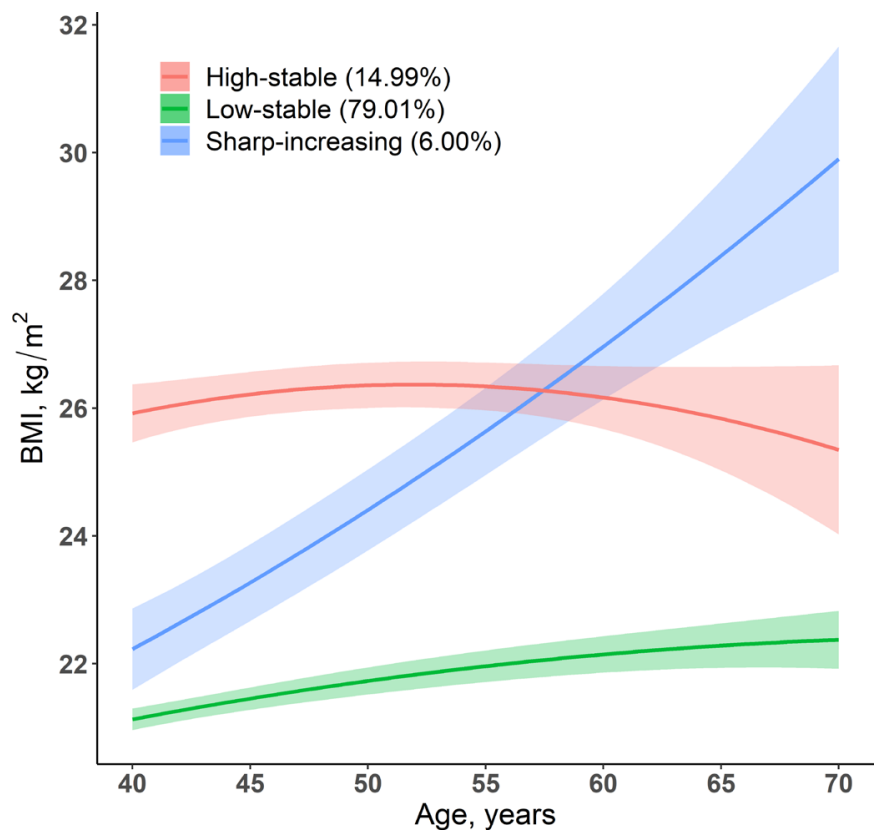

Figure 1 BMI trajectories of Chinese classes aged 40-70 years (the $95 \% \mathrm{Cl}$ is shown in shadow). BMI, body mass index. urban-rural residence, BMI, age, SBP and DBP among the three trajectory classes were observed at baseline $(\mathrm{p}<0.05)$.

Table 2 shows the results of Cox regression models: HRs $(95 \%$ CI) for the relation between BMI trajectory classification and incident hypertension. Compared with the reference (low-stable) class, the crude HRs (95\% CI) for the sharp-increasing class and the high-stable class were 1.264 (1.074 to 1.489 ) and 1.514 (1.340 to 1.712), respectively, in model 1 . With age and gender corrected, the HRs (95\% CI) for the sharp-increasing class and the high-stable class were 1.372 (1.164 to 1.617 ) and 1.726 (1.523 to 1.956$)$, respectively, in model 2. With age, gender, smoking, alcohol drinking, urban-rural residence, working status, marital status, and education level adjusted, DBP and SBP level, the HRs (95\% CI) for the sharp-increasing class and the high-stable class were 1.321 (1.119 to 1.559 ) and 1.504 (1.322 to 1.711 ), respectively, in model 4. All four Cox regression models showed that BMI trajectory classification was a significant factor in the incidence of hypertension.

In Cox regression model 4, age, alcohol drinking, DBP and SBP were significant risk factors for incident hypertension. The HRs (95\% CI) were 1.122 (1.003 to 1.255$)$, 1.014 (1.006 to 1.022) and 1.027 (1.021 to 1.032), respectively. Smoking, residence, working status, marital status and education level were not significant for incident hypertension.

Figure 2 shows the results of Cox regression models: HRs (95\% CI) of model-predicted BMI values and slopes for incident hypertension by ages, adjusted for gender, 
Table 2 HR $(95 \%$ Cl) of individual BMI trajectory classes for incident hypertension

\begin{tabular}{|c|c|c|c|c|}
\hline & Model 1 & Model 2 & Model 3 & Model 4 \\
\hline \multicolumn{5}{|l|}{ Trajectory classes } \\
\hline Low-stable & Reference & Reference & Reference & Reference \\
\hline High-stable & 1.514 (1.34 to 1.712$)$ & 1.726 (1.523 to 1.956$)$ & 1.733 (1.529 to 1.965$)$ & 1.504 (1.322 to 1.711$)$ \\
\hline \multicolumn{5}{|l|}{ Covariates } \\
\hline Age & & $1.052(1.044$ to 1.060$)$ & $1.052(1.044$ to 1.060$)$ & 1.047 (1.038 to 1.056$)$ \\
\hline Smoking & & & $0.979(0.861$ to 1.113$)$ & 0.972 (0.855 to 1.106$)$ \\
\hline Alcohol drinking & & & $1.089(0.976$ to 1.215$)$ & $1.122(1.003$ to 1.255$)$ \\
\hline Urban & & & & 1.083 (0.975 to 1.202$)$ \\
\hline Employed & & & & $0.882(0.772$ to 1.007$)$ \\
\hline Primary school or no & & & & Reference \\
\hline Middle school & & & & 1.094 (0.974 to 1.229$)$ \\
\hline High school or above & & & & 0.960 (0.839 to 1.099$)$ \\
\hline DBP & & & & 1.014 (1.006 to 1.022$)$ \\
\hline SBP & & & & 1.027 (1.021 to 1.032$)$ \\
\hline
\end{tabular}

Individual model-predicted BMI values, slopes and incident hypertension.

Model 1: unadjusted for any covariates.

Model 2: adjusted for gender and age.

Model 3: adjusted for gender, age, smoking and alcohol drinking.

Model 4: adjusted for gender, age, smoking, alcohol drinking, urban-rural residence, working status, marital status, education level, DBP and SBP level.

BMI, body mass index; DBP, diastolic blood pressure; SBP, systolic blood pressure.

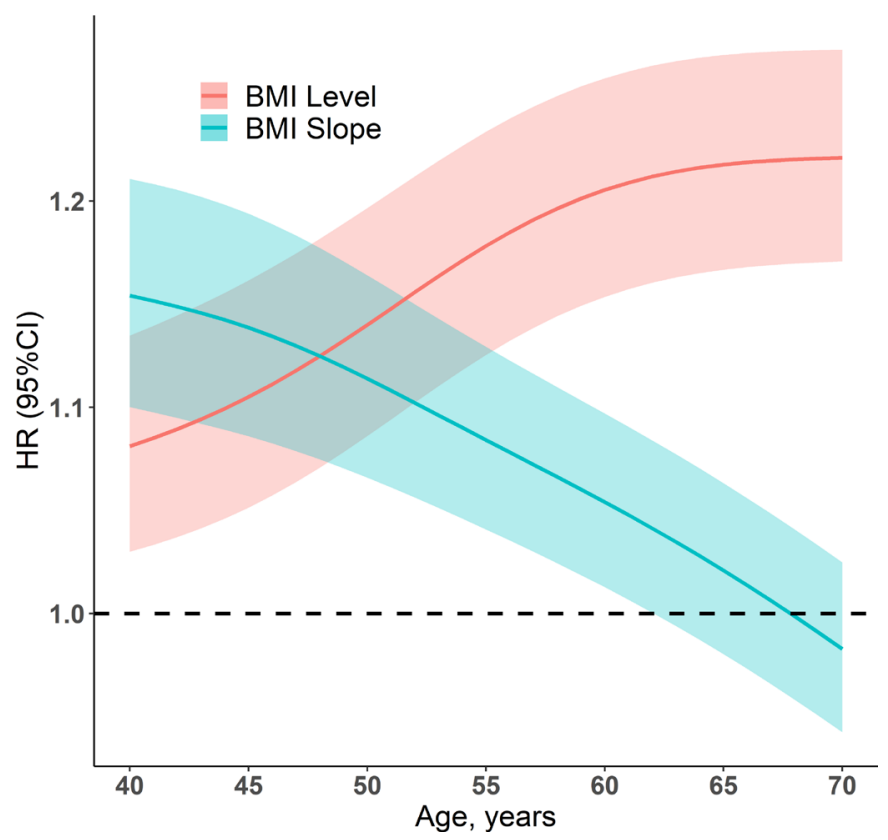

Figure 2 HRs $(95 \% \mathrm{Cl})$ of model-predicted BMI values and BMI slopes for incident hypertension by age, with adjustment for gender, smoking, alcohol drinking, working status, urbanrural residence, marital status and education level. The 95\% Cls are shown in shadow. BMI, body mass index. smoking, alcohol drinking, working status, urban-rural residence, marital status and education level. HRs $(95 \%$ CI) of BMI values increased rapidly during the age of 40-60 years before slowing down. The correlation between BMI values and the onset of hypertension is significantly positive for the class aged 40-70 years. HRs (95\% CI) of BMI values ranged from 1.081 (1.030 to 1.135 ) to 1.221 (1.171 to 1.273 ) (online supplemental table S4). HRs (95\% CI) of BMI slopes decreased for the age of 40-70 years and ranged from 0.983 (0.943 to 1.025$)$ to 1.154 (1.100 to 1.211$)$. No significant correlation between BMI slopes and incident hypertension was found for the age of 63-70 years. HRs of BMI slopes were higher than those of BMI values for the age of 40-47 years.

\section{DISCUSSION}

In this open cohort study, we observed three distinct individual BMI trajectory classes in their mid-life to elderly adulthood were closely associated with the onset of hypertension. Compared with the low-stable class, the sharpincreasing and the high-stable classes were confronted with more risk factors for incident hypertension. Furthermore, a significant correlation between model-predicted BMI slopes for the age between 40 and 62 years and 
incident hypertension was found (online supplemental table S4). Available evidence suggests that the change in $\mathrm{BMI}$ is indicative of the risk of hypertension. ${ }^{14}$ However, in most of the previous researches, no importance was attached to BMI values and slopes at distinct ages for predicting incident hypertension. This study explored the role of BMI values and slopes at distinct ages (40-70 years) in predicting hypertension risk in later life.

Three different individual BMI trajectory classes for Chinese aged 40-70 years were defined in this study. For the low-stable class, their BMI increased slowly and they kept normal weight. For the sharp-increasing class, their BMI increased rapidly and they developed into obesity eventually, while the high-stable class maintained a high BMI value and remained overweight. What's more, researchers have identified the trajectory of BMI in distinct populations over specific life periods, for British, Canadian and Chinese before. ${ }^{927}{ }^{28}$ Individual BMI trajectories were characterised by follow-up time or age in these researches. As far as we know, no studies have ever been done among Chinese aged 40-70 years and found the correlation between individual BMI trajectories and the incidence of hypertension.

As proved by the research, the high-stable class had the highest hypertension risk. Participants in the high-stable class had the highest BMI value for those aged 40-50 years. The crude HR $(95 \% \mathrm{CI})$ for the high-stable class was 1.514 (1.340 to 1.712). After the adjustment for smoking, alcohol drinking, urban-rural residence, working status, marital status, education level, DBP and SBP level, the HR $(95 \%$ CI) for the high-stable class was 1.504 (1.322 to 1.711 ), being still a big number. In the trajectory which persisted, high BMI value was found in our study to be a risk factor for incident hypertension, in agreement with previous research on the correlation between BMI values and hypertension. ${ }^{1429-32}$ In comparison with the low-stable class, the sharp-increasing class also showed a higher risk of incident hypertension and the adjusted HR (95\% CI) was 1.321 (1.119 to 1.559 ). Similar to previous studies, ${ }^{1433} 34$ a higher rate of increase in BMI was related to a higher risk of incident hypertension. Consistent with previous studies, ${ }^{35}{ }^{36}$ Cox regression model 4 indicated that age, alcohol drinking, DBP and SBP were significant risk factors for incident hypertension. However, gender, smoking, residence, working status, marital status and educational level were not found to be major factors that have an impact on the incident hypertension.

In this study, the HRs for individual model-predicted BMI values were larger than 1 and increased rapidly at first before turning to be stable. This indicated that BMI values were significant risk factors for hypertension, and the risk degree increased with age and stayed high after the age of 60 . The HRs of individual model-predicted BMI slopes were decreased for the age of 40-70 years. The slopes of BMI were also risk factors for hypertension (except for the age of 69 and 70 years), and the risk degree decreased with age. By analysing the HRs of model-predicted BMI values and slopes, we found the age of 40-47 years is a critical period, showing that participants whose HRs of BMI slopes were higher than those of BMI values during this period. As a result, BMI slopes were more important for predicting incident hypertension during 40-47 years old. Therefore, more attention should be paid to people whose BMI increased rapidly during the age of $40-47$ years. Previous studies ${ }^{142}$ had shown that the critical age period can be identified by BMI values and slopes, which are related to the incident disease. So far, there were no comparable data with our study on the correlation between BMI slopes and the risk of developing hypertension during the age of 40-70 years.

With LCGMM, individual model-predicted BMI values and slopes could be used to characterise the critical period to develop hypertension. ${ }^{27}$ On the basis of lifecourse epidemiology theory, exposure at a specific life period can greatly affect the development of diseases in later life. ${ }^{37}$ As a result, our study suggested that maintaining a high BMI value or a rapid increase in BMI may increase the risk of hypertension in later life.

This study had many advantages. First, we obtained data from a large national survey and used LCGMM to perform a longitudinal study, namely, LCGMM was applied to characterise different individual BMI trajectories and calculate BMI values and slopes. Second, three repeated measurements for DBP and SBP were taken under the same protocol. Nevertheless, this study was subject to some limitations. As some patients with hypertension were defined by self-report, some cases of incident hypertension may have been overlooked. We only included the normotensives at baseline, and consequently we may have underestimated the risk of hypertension for changes in BMI. We considered the influence of factors, such as the history of other hypertension-related diseases (like diabetes) and physical activities on the incidence of hypertension. However, we did not have enough relevant variables or too many values were missing in CHNS data (more than 30\% missing). For this reason, we cannot eliminate the possibility of confounding by those factors. As the data were collected among Chinese participants, the findings might not apply to other ethnic classes.

\section{CONCLUSION}

In this study, a substantive correlation was found between distinct individual BMI trajectory classes and the incidence of hypertension. Compared with the low-stable class, sharp-increasing class and high-stable class are confronted with significant risk factors for incident hypertension. Individual BMI slope is an important indicator of incident hypertension. The period of ageing from 40 to 47 years is critical for incident hypertension. In this regard, more effective public health interventions should be given to middle-aged and elderly people for the early prevention of hypertension, especially those with rapid weight gains or keeping high BMI values. 
Acknowledgements This research uses data from China Health and Nutrition Survey (CHNS). We are so grateful to the National Institute for Nutrition and Health, the China Center for Disease Control and Prevention, Carolina Population Center (P2C HD050924, T32 HD007168), the University of North Carolina at Chapel Hill, the National Institutes of Health (NIH) (R01-HD30880, DK056350, R24 HD050924 and R01-HD38700) and the NIH Fogarty International Center (D43 TW009077, D43 TW007709) for their financial support in the CHNS data collection and future surveys, and for analysis files from 1989 to 2015 . We are also thankful to the China-Japan Friendship Hospital and the Ministry of Health for the support of CHNS 2009. At last, we also appreciate the Chinese National Human Genome Center at Shanghai and Beijing Municipal Center for Disease Prevention and Control for their commitment since 2009 and 2011, respectively.

Contributors Conceptualisation: QiZ and QingZ. Data curation: QiZ. Methodology and software: QiZ and QingZ. Validation: QingZ. Formal analysis: QiZ. Resources: QiZ. Writing —original draft preparation: QiZ. Writing—review and editing: QiZ and QingZ. Visualisation: QiZ and LS. Supervision: QingZ. Project administration: QingZ. All authors have checked and proofread the final version of the manuscript.

Funding The authors have not declared a specific grant for this research from any funding agency in the public, commercial or not-for-profit sectors.

Competing interests None declared.

Patient consent for publication Not required.

Provenance and peer review Not commissioned; externally peer-reviewed.

Data availability statement Data are available in a public, open access repository. The data sets generated and/or analysed during the current study are available in the web: https://www.cpc.unc.edu/projects/china.

Supplemental material This content has been supplied by the author(s). It has not been vetted by BMJ Publishing Group Limited (BMJ) and may not have been peer-reviewed. Any opinions or recommendations discussed are solely those of the author(s) and are not endorsed by BMJ. BMJ disclaims all liability and responsibility arising from any reliance placed on the content. Where the content includes any translated material, BMJ does not warrant the accuracy and reliability of the translations (including but not limited to local regulations, clinical guidelines, terminology, drug names and drug dosages), and is not responsible for any error and/or omissions arising from translation and adaptation or otherwise.

Open access This is an open access article distributed in accordance with the Creative Commons Attribution Non Commercial (CC BY-NC 4.0) license, which permits others to distribute, remix, adapt, build upon this work non-commercially, and license their derivative works on different terms, provided the original work is properly cited, appropriate credit is given, any changes made indicated, and the use is non-commercial. See: http://creativecommons.org/licenses/by-nc/4.0/.

ORCID iD

Qing Zeng http://orcid.org/0000-0001-6132-7398

\section{REFERENCES}

1 Wang Z, Chen Z, Zhang L, et al. Status of hypertension in China: results from the China hypertension survey, 2012-2015. Circulation 2018; 137:2344-56.

2 Li Y, Yang L, Wang L, et al. Burden of hypertension in China: a nationally representative survey of 174,621 adults. Int $J$ Cardiol 2017;227:516-23.

3 Lu J, Lu Y, Wang X, et al. Prevalence, awareness, treatment, and control of hypertension in China: data from 1.7 million adults in a population-based screening study (China peace million persons project). The Lancet 2017;390:2549-58.

4 Hall JE, do Carmo JM, da Silva AA, et al. Obesity-Induced hypertension: interaction of neurohumoral and renal mechanisms. Circ Res 2015;116:991-1006.

5 Seravalle G, Grassi G. Obesity and hypertension. Pharmacol Res 2017;122:1-7.

6 Jiang S-Z, Lu W, Zong X-F, et al. Obesity and hypertension. Exp Ther Med 2016;12:2395-9.

7 Zhao Y, Liu Y, Sun H, et al. Association of long-term dynamic change in body weight and incident hypertension: the rural Chinese cohort study. Nutrition 2018;54:76-82.
8 Hossain FB, Adhikary G, Chowdhury AB, et al. Association between body mass index (BMI) and hypertension in South Asian population: evidence from nationally-representative surveys. Clin Hypertens 2019;25:28.

9 Tu AW, Mâsse LC, Lear SA, et al. Body mass index trajectories from ages 1 to 20: results from two nationally representative Canadian longitudinal cohorts. Obesity 2015;23:1703-11.

10 Wang X, Dong B, Huang S, et al. Body mass index trajectory and incident hypertension: results from a longitudinal cohort of Chinese children and adolescents, 2006-2016. Am J Public Health 2020;110:1689-95.

11 Buscot M-J, Thomson RJ, Juonala M, et al. Distinct child-to-adult body mass index trajectories are associated with different levels of adult cardiometabolic risk. Eur Heart J 2018;39:2263-70.

12 Munthali RJ, Kagura J, Lombard Z, et al. Childhood adiposity trajectories are associated with late adolescent blood pressure: birth to twenty cohort. BMC Public Health 2016;16:665.

13 Boyer BP, Nelson JA, Holub SC. Childhood body mass index trajectories predicting cardiovascular risk in adolescence. J Adolesc Health 2015;56:599-605.

14 Fan B, Yang Y, Dayimu A, et al. Body mass index trajectories during young adulthood and incident hypertension: a longitudinal cohort in Chinese population. J Am Heart Assoc 2019;8:e011937.

15 Yuan Y, Chu C, Zheng W-L, et al. Body mass index trajectories in early life is predictive of cardiometabolic risk. $J$ Pediatr 2020;219:31-7.

16 Jiao Y, Sun Z, Xie Y, et al. Potential impacts of the 2017 American College of Cardiology/American heart association high blood pressure guideline on Chinese adults and how to address them. BMC Cardiovasc Disord 2020;20:237.

17 Chen Y, Peng Q, Yang Y, et al. The prevalence and increasing trends of overweight, general obesity, and abdominal obesity among Chinese adults: a repeated cross-sectional study. BMC Public Health 2019;19:1293.

18 Thomas U, Claudio B, Fadi C. International Society of hypertension global hypertension practice guidelines. Hypertension 2020;2020:1334-57.

19 Wang EA, Pletcher M, Lin F, et al. Incarceration, incident hypertension, and access to health care: findings from the coronary artery risk development in young adults (cardia) study. Arch Intern Med 2009;169:687.

20 Galbraith S, Bowden J, Mander A. Accelerated longitudinal designs: an overview of modelling, power, costs and handling missing data. Stat Methods Med Res 2017;26:374-98.

21 Duncan SC, Duncan TE, Hops H. Analysis of longitudinal data within accelerated longitudinal designs. Psychol Methods 1996;1:236-48.

22 Zhang B, Zhai FY, Du SF, et al. The China health and nutrition survey, 1989-2011. Obes Rev 2014;15 Suppl 1:2-7.

23 Zhou B-F, Cooperative Meta-Analysis Group of the Working Group on Obesity in China. Predictive values of body mass index and waist circumference for risk factors of certain related diseases in Chinese adults--study on optimal cut-off points of body mass index and waist circumference in Chinese adults. Biomed Environ Sci 2002;15:83-96.

24 Wang H, Du S, Zhai F, et al. Trends in the distribution of body mass index among Chinese adults, aged $20-45$ years (1989-2000). Int J Obes 2007;31:272-8.

25 Proust-Lima C, Philipps V, Liquet B. Estimation of Extended Mixed Models Using Latent Classes and Latent Processes: The $R$ Package Icmm. J Stat Softw 2017;78.

26 Yuan Z, Yang Y, Wang C, et al. Trajectories of Long-Term normal fasting plasma glucose and risk of coronary heart disease: a prospective cohort study. J Am Heart Assoc 2018;7.

27 Lv J, Fan B, Wei M, et al. Trajectories of early to mid-life adulthood $\mathrm{BMI}$ and incident diabetes: the China health and nutrition survey. BMJ Open Diabetes Res Care 2020;8:e000972.

28 Sperrin M, Marshall AD, Higgins V, et al. Slowing down of adult body mass index trend increases in England: a latent class analysis of cross-sectional surveys (1992-2010). Int J Obes 2014;38:818-24.

29 Tsujimoto T, Sairenchi T, Iso H, et al. Impact of obesity on incident hypertension independent of weight gain among nonhypertensive Japanese: the Ibaraki Prefectural health study (IPHS). J Hypertens 2012;30:1122-8.

30 Channanath AM, Farran B, Behbehani K, et al. Association between body mass index and onset of hypertension in men and women with and without diabetes: a cross-sectional study using National health data from the state of Kuwait in the Arabian Peninsula. BMJ Open 2015;5:e007043.

31 Matsuo T, Sairenchi T, Suzuki K, et al. Long-Term stable obesity increases risk of hypertension. Int J Obes 2011;35:1056-62.

32 Roka R, Michimi A, Macy G. Associations between hypertension and body mass index and waist circumference in U. S. Adults: A 
Comparative Analysis by Gender. High Blood Press Cardiovasc Prev 2015;22:265-73.

33 Zhang M, Zhao Y, Sun $\mathrm{H}$, et al. Effect of dynamic change in body mass index on the risk of hypertension: results from the rural Chinese cohort study. Int J Cardiol 2017;238:117-22.

34 Uchiyama A, Shimizu T, Nakagawa T, et al. Association of hypertension with changes in the body mass index of university students. Environ Health Prev Med 2008;13:271-80.
35 Echouffo-Tcheugui JB, Batty GD, Kivimäki M, et al. Risk models to predict hypertension: a systematic review. PLoS One 2013;8:e67370.

36 Roerecke M, Kaczorowski J, Tobe SW, et al. The effect of a reduction in alcohol consumption on blood pressure: a systematic review and meta-analysis. Lancet Public Health 2017;2:e108-20.

37 Lynch J, Smith GD. A life course approach to chronic disease epidemiology. Annu Rev Public Health 2005;26:1-35. 